Physical Picture of the Electromagnetic Fields between Two Infinite Conducting Plates Produced by a Point Charge Moving at the Speed of Light

\title{
Introduction
}

There have been many papers since the original article by Condon on the fields that are produced when a bunch of charge passes through a cavity.' Many of these papers deal only with the energy loss by the bunch and hence are interested in the energy left in the cavity after the bunch is no longer in the cavity. ${ }^{2,3,4}$ For these purposes, one usually uses an unretarded coulomb gauge and it is only necessary to calculate the vector potential produced by the beam. However, if one is interested in the effect upon the bunch due to short-range fields produced by the bunch, one must also include the scaler potential in deriving the fields.

In the study of beam-cavity coupling effects, one must solve Maxwell's equations for the fields produced by a given beam shape and given cavity geometry. A recent paper that treats the effect on the bunch shape has considered the longitudinal electric field in a pill box cavity produced by a step function charge pulse traveling at the speed of 1 ight. ${ }^{5}$ In order to obtain a clear physical picture of how the fields are produced in the cavity, we treat the problem of a point charge traveling at the speed of light, $c$, between two infinite plates. This must, of course, give the same result as the closed pill box cavity for values of time $t$ such that cit is less than the cavity radius.

In the first section, the longitudinal and radial electric field components and the azimuthal magnetic field component are derived from Maxwell's equations for this idealized case. We use the eigenmode expansion method and include some details of the tricks used in the computation of the sums. In the second section, we discuss the physical picture of the electromagnetic fields that were derived in the previous section. 


\section{Field Computation}

We want to find the fields between two infinite perfectly-conducting plates located at $z=0$ and $z=g$, as shown in Fig. 1, produced by the point charge source

$$
\begin{aligned}
& \rho= \begin{cases}Q \delta(x) \delta(y) \delta(z-c t), & \text { if } 0<c t<g \\
0, & \text { otherwise }\end{cases} \\
& \vec{j}=\rho c \hat{z},
\end{aligned}
$$

with $\delta(x)$ the Dirac delta-function.

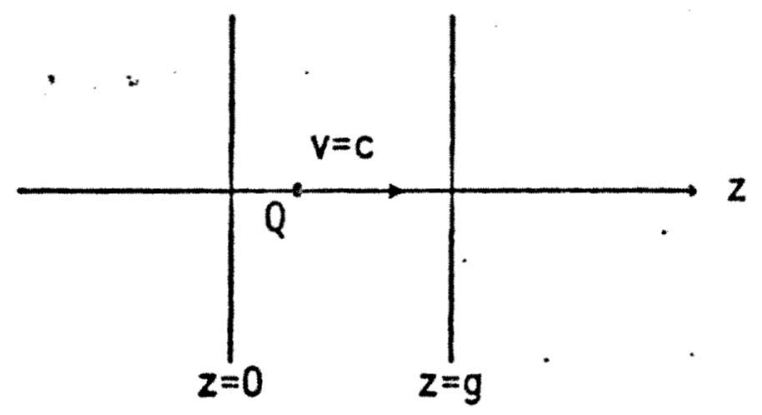

Fig. 1

We look for one eigenmode of the potential specified by $A_{\lambda}$ and $\phi_{\lambda}$ which satisfy the following conditions:

$$
\begin{aligned}
& \nabla^{2} \vec{A}_{\lambda}+\frac{\omega_{\lambda}^{2}}{c^{2}} \vec{A}_{\lambda}=0 \text {. } \\
& \nabla^{2} \phi_{\lambda}+\frac{\omega_{\lambda}^{2}}{c^{2}} \phi_{\lambda}=0 \\
& \left.\begin{array}{l}
\left(A_{\lambda}\right)_{x, y}=0 \\
\frac{\partial \phi_{\lambda}}{\partial z}=0
\end{array}\right\} \text { on plates, }
\end{aligned}
$$

where the parameter $\lambda$-identifies the eigenmode and stands for the three 
mode numbers:

$$
\lambda \equiv\left(k_{x}, k_{y}, m\right)
$$

We use the following normalization conditions:

$$
\int_{\text {cavity }} \vec{A}_{\lambda} \cdot \vec{A}_{\lambda^{\prime}}^{*} d^{3} r=\int_{\text {cavity }} \phi_{\lambda} \phi_{\lambda^{\prime}}^{*} d^{3} r=\delta\left(k_{x}-k_{x}^{\prime}\right) \delta\left(k_{y}-k_{y}^{\prime}\right) \delta_{m, m^{\prime}} .
$$

With the above definitions, we find that

$$
\begin{aligned}
& \left(A_{\lambda}\right)_{x}=\frac{-i m k_{x} c}{g \omega_{\lambda} \sqrt{2 g\left(k_{x}^{2}+k_{y}^{2}\right)}} e^{i\left(k_{x} x+k_{y} y\right)} \sin \frac{m \pi}{g} \\
& \left(A_{\lambda}\right)_{y}=\frac{-i m k_{y} c}{g \omega_{\lambda} \sqrt{2 g\left(k_{x}^{2}+k_{y}^{2}\right)}} e^{i\left(k_{x} x+k_{y} y\right)} \sin \frac{m \pi}{g} \\
& \left(A_{\lambda}\right)_{z}=\frac{c}{\pi \omega_{\lambda}} \sqrt{\frac{P_{m}\left(k_{x}^{2}+k_{y}^{2}\right)}{2 g}} e^{i\left(k_{x} x+k_{y} y\right)} \cos \frac{m \pi z}{g}
\end{aligned}
$$

and

$$
\phi_{\lambda}=\frac{1}{\pi \sqrt{2 g}} e^{i\left(k_{x} x+k_{y} y\right)} \sin \frac{m \pi z}{g}
$$

with

$$
P_{m}=\left\{\begin{array}{l}
1 \text { if } m \neq 0 \\
\frac{3}{2} \text { if } m=0
\end{array}\right.
$$

The mode numbers $k_{x}$ and $k_{y}$ are real to assure that the potential $\vec{A}_{\lambda}$ and $\phi_{\lambda}$ are bounded.

It will be useful to introduce the following notation:

$$
s_{\lambda}=\int_{-\infty}^{\infty} d k \int_{-\infty}^{\infty} d k \sum_{m=0}^{\infty} \text {. }
$$

We decompose the $\underline{\underline{A}}$ and $\Phi$ into the eigenmode components as follows:

$$
\begin{aligned}
& \overrightarrow{\vec{A}}=s_{\lambda} q_{\lambda}(t) \vec{A}_{\lambda}(\vec{r}) \\
& \Phi=s_{\lambda} r_{\lambda}(t) \phi_{\lambda}(\vec{r}) .
\end{aligned}
$$


Then Maxwell's equations become

$$
\ddot{q}_{\lambda}+\omega_{\lambda}^{2} q_{\lambda}=4 \pi c \int_{\text {cavity }} \vec{J} \cdot \vec{A}_{\lambda}^{\star} d^{3} r
$$

and

$$
r_{\lambda}=\frac{4 \pi c^{2}}{\omega_{\lambda}^{2}} \int_{\text {cavity }} \rho \phi_{\lambda}^{*} d^{3} r .
$$

For the case when $\rho$ and $\vec{J}$ are given by Eq. (1), the solutions for $q_{\lambda}(t)$ and $r_{\lambda}(s)$, for $t \geq 0$, are given by

$$
r_{\lambda}= \begin{cases}\frac{4 c^{2} Q}{\omega_{\lambda}^{2} \sqrt{2 g}} \sin \frac{m \pi c t}{g} & \text { for } 0<c t<g \\ 0 & \text { for } c t>g .\end{cases}
$$

$$
\text { and } q_{\lambda}= \begin{cases}\frac{4 c Q}{k \omega_{\lambda}} \sqrt{\frac{P_{m}}{2 g}}\left[\cos \frac{m \pi c t}{g}-\cos \omega_{\lambda} t\right] & \text { for } 0<c t<g \\ \frac{4 c Q}{k \omega_{\lambda}} \sqrt{\frac{P_{m}}{2 g}}\left[-\cos \omega_{\lambda} t+(-1)^{m} \cos \left(\omega_{\lambda} t-\omega_{\lambda} g / c\right)\right] & \text { for ct }>g,\end{cases}
$$

with $k^{2}=k_{x}^{2}+k_{y}^{2}$. From $r_{\lambda}(t)$ and $q_{\lambda}(t)$, the fields can be obtained by

$$
\begin{aligned}
& \vec{E}=-s_{\lambda}\left[r_{\lambda} \nabla \phi_{\lambda}\right]-\frac{1}{c} s_{\lambda}\left[\dot{q}_{\lambda} \vec{A}_{\lambda}\right] . \\
& \vec{B}=s_{\lambda}\left[q_{\lambda}\left(\nabla \times \vec{A}_{\lambda}\right)\right] .
\end{aligned}
$$

Only three components of $\vec{E}$ and $\vec{B}$ do not vanish, namely: $E_{z}, E_{r}$ and $B_{\theta}$. In the following we derive each component separately. While we do not consider the fields at the exact positions of the point charge or at the plates, we can consider the fields for points infinitesimally close to these positions. 


\section{A. Longitudinal Electric Field}

The evaluation of $E_{z}$ is straightforward and yields

for $0<c t<g$;

$$
E_{z}=-\frac{2 Q}{\sqrt{c^{2} t^{2}-r^{2}}} \delta\left(z-\sqrt{c^{2} t^{2}-r^{2}}\right) H(c t-r)
$$

and for ct $>\mathrm{g}$,

$$
\begin{gathered}
E_{z}=-\frac{2 Q H(c t-r)}{\sqrt{c^{2} t^{2}-r^{2}}} \sum_{k=-\infty}^{\infty}\left\{\delta\left(z-\sqrt{c^{2} t^{2}-r^{2}}+2 k g\right)+\delta\left(z+\sqrt{c^{2} t^{2}-r^{2}}-2 k g\right)\right. \\
+\frac{2 Q H(c t-g-r)}{\sqrt{(c t-g)^{2}-r^{2}}} \sum_{k=-\infty}^{\infty}\left\{\delta\left[z-\sqrt{(c t-g)^{2}-r^{2}}+(2 k+1) g\right]\right. \\
\left.\quad+\delta\left[z+\sqrt{(c t-g)^{2}-r^{2}}-(2 k+1) g\right]\right\}
\end{gathered}
$$

where $r^{2}=x^{2}+y^{2}$. Note that the above expressions for $E_{z}$ are valid even for $r=0$.

In obtaining Eqs. (8) and (9), we have used the following integral re-

\section{lation:}

$\int_{0}^{\infty} d x\left\{x J_{0}(c x) \frac{\sin \left[a \sqrt{b^{2}+x^{2}}\right]}{\sqrt{b^{2}+x^{2}}}\right\}=\operatorname{sgn}(a) H(|a|-|c|) \frac{\cos \cdot\left[|b| \sqrt{a^{2}-c^{2}}\right]}{\sqrt{a^{2}-c^{2}}}$

B. Radial Electric Field

The field $E_{r}$ may be obtained from a potential function $V(r)$ by means of

where

$$
E_{r}=-\frac{\partial V(r)}{\partial r} \text {, }
$$

$$
V(r)=\frac{2 Q c}{g^{2}} s_{\lambda} e^{i\left(k_{x} x+k_{y} y\right) \frac{m}{k^{2}} \sin \frac{m \pi z}{g}}\left\{\begin{array}{l}
\frac{g}{m \pi c} \sin \frac{m \pi c t}{g}-\frac{1}{\omega_{\lambda}} \sin \omega_{\lambda} t, \\
\text { if } 0<c t<g ; \\
\frac{(-1)^{m}}{\omega_{\lambda}} \sin \omega_{\lambda}\left(t-\frac{g}{c}-\frac{1}{\omega_{\lambda}} \sin \omega_{\lambda} t,\right. \\
\text { if } c t>g .
\end{array}\right.
$$


To demonstrate how to perform the sums to calculate $E_{r}$, we have worked out the case when $0<c t<g$ in full detail. Similar methods apply when $c t>g$.

For $0<c t<g$, we have

$$
\begin{aligned}
\frac{\partial V}{\partial t} & =\frac{2 Q c}{g^{2}} s_{\lambda}\left\{e^{i\left(k_{x} x+k_{y} y\right)} \sin \frac{m z}{g} \cdot \frac{m}{k^{2}}\left[\cos \frac{m \pi c t}{g}-\cos \omega_{\lambda} t\right]\right\} \\
& =\frac{2 Q c}{g^{2}} s_{\lambda}\left\{e^{i\left(k_{x} x+k_{y} y\right)} \sin \frac{m \pi z}{g} \cdot \frac{m}{k^{2}} \int_{0}^{k} d \xi \frac{c t \xi \sin \left[c t \sqrt{\xi^{2}+\left(\frac{m \pi}{g}\right)^{2}}\right]}{\sqrt{\xi^{2}+\left(\frac{m \pi}{g}\right)^{2}}}\right\} \\
& =\frac{4 \pi Q c}{g^{2}} \sum_{m=1}^{\infty} \int_{0}^{\infty} k d k J_{0}(k r) \sin \frac{m z}{g} \cdot \frac{m}{k^{2}} \int_{0}^{k} d \xi \frac{c t \xi \sin \left[c t \sqrt{\xi^{2}+\left(\frac{m \pi}{g}\right)^{2}}\right]}{\sqrt{\xi^{2}+\left(\frac{m \pi}{g}\right)^{2}}} .
\end{aligned}
$$

We now switch the order of integration, using

$$
\int_{0}^{\infty} d k \int_{0}^{k} d \xi \ldots=\int_{0}^{\infty} d \xi \int_{\xi}^{\infty} d k \ldots,
$$

$\frac{\partial V}{\partial t}=\frac{4 \pi Q c}{g^{2}} \sum_{m=1}^{\infty} m \sin \frac{m \pi z}{g} \int_{0}^{\infty} d \xi \frac{c t \xi \sin \left[c t \sqrt{\xi^{2}+\left(\frac{m}{g}\right)^{2}}\right]}{\sqrt{\xi^{2}+\left(\frac{m \pi}{g}\right)^{2}}} \int_{\xi}^{\infty} \frac{d k}{k} J_{Q}(k r)$.

Therefore,

$$
\begin{aligned}
-\frac{\partial E_{r}}{\partial t} & =\frac{\partial^{2} V}{\partial t \partial r} \\
& =-\frac{4 \pi Q c}{g^{2}} \sum_{m=1}^{\infty} m \sin \frac{m \pi}{g} \int_{0}^{\infty} d \xi \frac{c t \xi \sin \left[c t \sqrt{\xi^{2}+\left(\frac{m \pi}{g}\right)^{2}} \int_{\xi}^{\infty} d k J_{1}(k r)\right.}{\sqrt{\xi^{2}+\left(\frac{m \pi}{g}\right)^{2}}} \\
& = \begin{cases}0 \text { if } r=0 & 4 \pi Q c \\
-\frac{4 \pi}{g^{2}} \sum_{m=1}^{\infty} m \sin \frac{m z}{g} \int_{0}^{\infty} d \xi \frac{c t \xi \sin \left[c t \sqrt{\xi^{2}+\left(\frac{m \pi}{g}\right)^{2}}\right]}{\sqrt{\xi^{2}+\left(\frac{m \pi}{g}\right)^{2}}} \frac{J_{0}(\xi r)}{r} & \text { if } r \neq 0 .\end{cases}
\end{aligned}
$$


For $r \neq 0$, we then use Eq. (10) to obtain

$$
\frac{\partial E_{r}}{\partial t}=\frac{4 \pi Q c}{g^{2}} \sum_{m=1}^{\infty} m \sin \frac{m \pi z}{g} \frac{c t H(c t-r)}{r \sqrt{c^{2} t^{2}-r^{2}}} \cos \left[\frac{m \pi}{g} \sqrt{c^{2} t^{2}-r^{2}}\right] \text {. }
$$

Integrating both sides with respect to $t$ from 0 to $t$, we find

$$
\begin{aligned}
E_{r} & =\frac{4 Q}{g r} H(c t-r) \sum_{m=1}^{\infty} \sin \frac{m \pi z}{g} \sin \left[\frac{m \pi}{g} \sqrt{c^{2} t^{2}-r^{2}}\right] \\
& =\frac{2 Q}{r} H(c t-r) \delta\left(z-\sqrt{c^{2} t^{2}-r^{2}}\right), \quad \text { for }\left\{\begin{array}{l}
0<r \\
0<z<g \\
0<c t<g .
\end{array}\right.
\end{aligned}
$$

By similar manipulation, we find for $E_{r}$ when $c t>g$,

$$
\begin{aligned}
E_{r} & =\frac{2 Q}{r} H(c t-r) \sum_{k=-\infty}^{\infty}\left\{\delta\left[z-\sqrt{c^{2} t^{2}-r^{2}}+2 k g\right]-\delta\left[z+\sqrt{c^{2} t^{2}-r^{2}}-2 k g\right]\right\} \\
& -\frac{2 Q}{r} H(c t-r-g) \sum_{k=-\infty}^{\infty}\left\{\delta\left[z-\sqrt{(c t-g)^{2}-r^{2}}+(2 k+1) g\right]\right. \\
& \left.-\delta\left[z+\sqrt{(c t-g)^{2}-r^{2}}-(2 k+1) g\right]\right\} .
\end{aligned}
$$

\section{Azimuthal Magnetic Field}

Having obtained expressions for the electric field, the easiest way to find the magnetic field is to proceed directly from Maxwell's equations instead of using Eq. (7). Away from $r=0$, the result is,

for $0<c t<g$ :

$$
B_{\theta}=\frac{2 Q c t}{r \sqrt{c^{2} t^{2}-r^{2}}} H(c t-r) \delta\left(z-\sqrt{c^{2} t^{2}-r^{2}}\right),
$$

and for $c t>g$, 


$$
\begin{gathered}
B_{\theta}=\frac{2 Q c t H(c t-r)}{r \sqrt{c^{2} t^{2}-r^{2}}} \sum_{k=-\infty}^{\infty}\left\{\delta\left[z+\sqrt{c^{2} t^{2}-r^{2}}+2 k g\right]+\delta\left[z-\sqrt{c^{2} t^{2}-r^{2}}-2 k g\right]\right\} \\
-\frac{2 Q(c t-g) H(c t-g-r)}{r \sqrt{c^{2} t^{2}-r^{2}}} \sum_{k=-\infty}^{\infty}\left\{\delta\left[z+\sqrt{(c t-g)^{2}-r^{2}}+(2 k+1) g\right]\right. \\
\left.+\delta\left[z-\sqrt{(c t-g)^{2}-r^{2}}-(2 k+1) g\right]\right\} .
\end{gathered}
$$

\section{The Physical Picture}

As the point charge $Q$ enters the cavity, charges and currents are induced on the first conducting plate. These charges move out radially at the speed of light and are on a circle of radius $r=c t$. The total charge on the plate is $-Q$. The field due to these induced charges and currents plus the field due to the point charge and current is a $\delta$-function signal on the surface of the light sphere, $c^{2} t^{2}=r^{2}+z^{2}$. See Fig. $2 a$.

The electric field $\vec{E}$ is tangential to the surface, while the Poynting vector $\vec{S}$ is perpendicular to the surface. The magnetic field is along $\hat{\theta}$ direction and satisfies $|\vec{B}|=|\vec{E}|$. Notice that no field exists inside the sphere. This should not be surprising because, in free space, an uitra-relativistic particle does not deposit any field along its way. It only produces a field in the plane which contains the particle and is perpendicular to its direction of motion. Indeed, in our case, if we remove the second plate, and let

$$
\begin{aligned}
t & +\infty \\
z & \rightarrow \infty \\
z-c t & + \text { finite } \\
r & + \text { finite }
\end{aligned}
$$

we obtain

$$
\begin{aligned}
& E_{z}+0 \\
& E_{r}=B_{\theta}+\frac{2 Q}{r} \delta(z-c t),
\end{aligned}
$$

which coincides with the case of a point charge moving at the speed of light in free space.

At time $c t=g$, both the charge and the spherical wave front of the field 
arrive at the second plate. At this time, drastic changes occur at the second plate. The net result is two sets of induced charges and currents. One set, with total charge $2 Q$, travels out radially on a circle $r^{2}=\left(c^{2} t^{2}-g^{2}\right)$ (with an apparent speed greater than the speed of light) and produces a reflection of the wave which reverses the sign of $E_{r}$ but retains the signs of $E_{z}$ and $B_{\theta}$.

The second set of induced charges and currents, with a total charge of $-Q$, travels out on a circle $r=(c t-g)$ at the speed of light. The field due to this second set is a $\delta$-function signal on a new light sphere $(c t-g)^{2}=r^{2}+(z-g)^{2}$. See Fig. $2 b$.

At time $c t=2 g$, both of these two wavefronts return to. the first plate and produce two new sets of induced charges and currents on the plate, one with total charge $-2 Q$ and the other with total charge $2 Q$. These induced sources produce a reflection of the waves, again reversing the sign of $E_{r}$ but retaining the signs of $E_{z}$ and $B_{\theta}$. This process is repeated at every interval defined by $c t=m g$ with $m$ an integer. For $m>2$, the two already existing sets of $\delta$-function waves are reflected back and forth between the plates. A picture of this process is shown in Fig. $2 a-c$ for three different time intervals.

It is interesting to note that a test charge traveling behind the source charge along the $z$ axis will not experience any force from the longitudinal electric field until a time after the source charge has passed through the second plate. From Eq. (9), we see that for a positive charge source at $r=0$ and ct $>g, E_{z}$ is positive, so that in the case of a positive test charge following a positive source charge, the test charge always gains energy from the wake field.

For the case when the test charge following the source charge is radially displaced from the $z$ axis, the test charge can receive several longitudinal and transverse impulses from the electromagnetic wake field. These effects will be more thoroughly discussed in a succeeding paper.

\section{Acknowledgements}

It is a pleasure to thank our colleagues E. Keil, R. H. Helm, M. J. Lee, J. R. Rees, H. Wiedemann and P. B. Wilson for many informative discussions. 


\section{References}

1. E. U. Condon, J. Appl. Phys. 12, 129 (1941).

2. V. K. Nei1, UCRL-9124 (1960), Thesis.

3. 0. A. Kolpakov and V. I. Kotov, Soviet Phys. Tech. Phys. 9, 1072 (1965).

4. E. Keil, Nucl. Instr. and Meth. 100, 419 (1972) and references therein.

5. A. Papiernik, M. Chatard-Moul in and B. Jecko, Proc. IX Int. Conf. on High Energy Acc., SLAC, 375 (1974). 


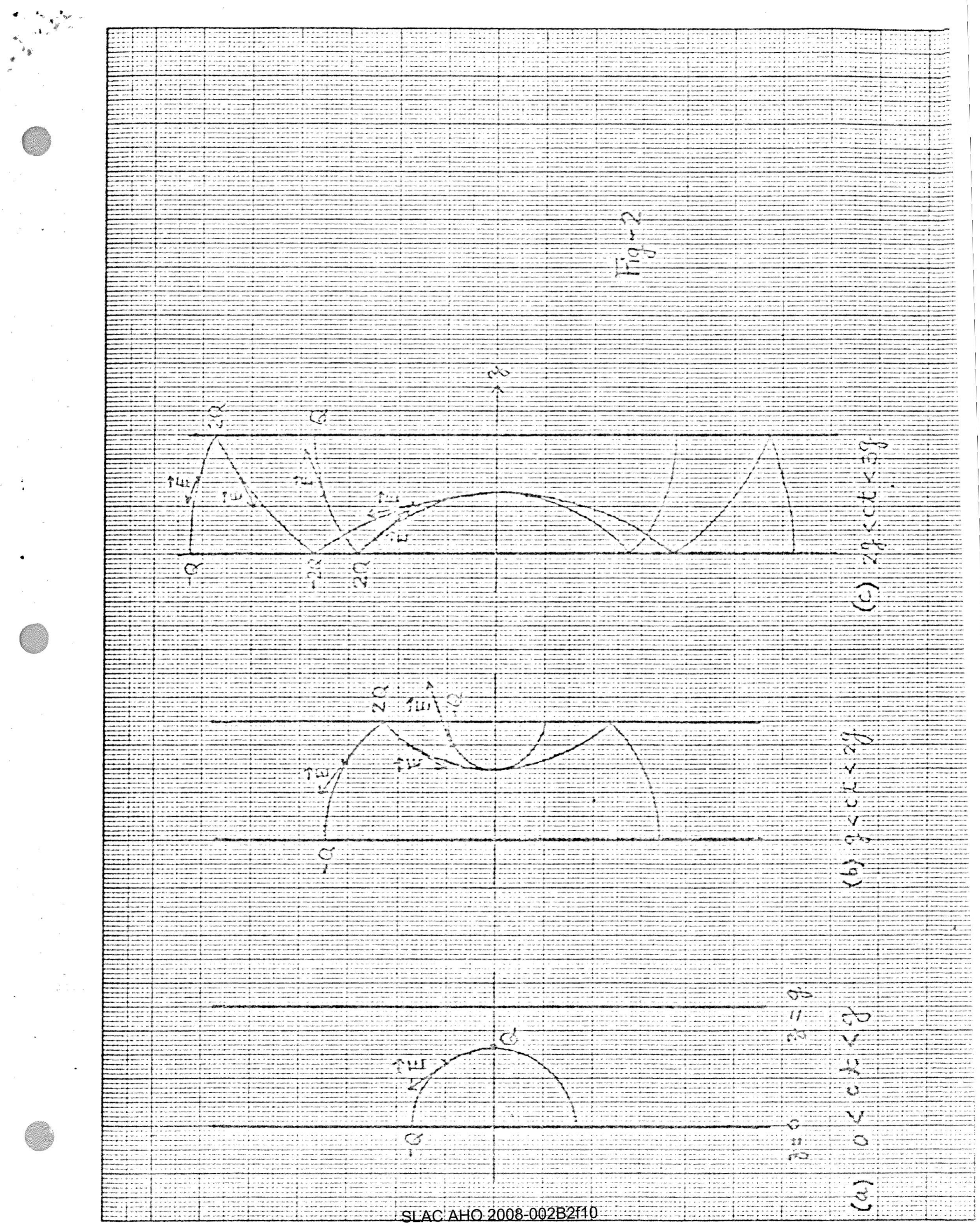

